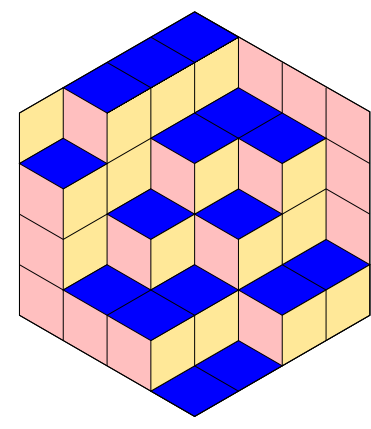

Richard Ehrenborg, Sophie Morel \& Margaret Readdy

Some combinatorial identities appearing in the calculation of the cohomology of Siegel modular varieties

Volume 2, issue 5 (2019), p. 863-878.

<http://alco.centre-mersenne.org/item/ALCO_2019__2_5_863_0>

(C) The journal and the authors, 2019.

Some rights reserved.

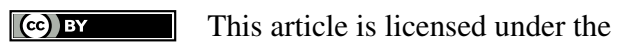

Creative Commons AtTRibution 4.0 International License.

http://creativecommons.org/licenses/by/4.0/

Access to articles published by the journal Algebraic Combinatorics on the website http://alco.centre-mersenne.org/ implies agreement with the Terms of Use (http://alco.centre- mersenne.org/legal/).

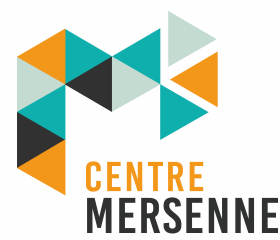

Algebraic Combinatorics is member of the Centre Mersenne for Open Scientific Publishing www.centre-mersenne.org 


\title{
Some combinatorial identities appearing in the calculation of the cohomology of Siegel modular varieties
}

\author{
Richard Ehrenborg, Sophie Morel \& Margaret Readdy
}

\begin{abstract}
In the computation of the intersection cohomology of Shimura varieties, or of the $L^{2}$ cohomology of equal rank locally symmetric spaces, combinatorial identities involving averaged discrete series characters of real reductive groups play a large technical role. These identities can become very complicated and are not always well-understood (see for example the appendix of [8]). We propose a geometric approach to these identities in the case of Siegel modular varieties using the combinatorial properties of the Coxeter complex of the symmetric group. Apart from some introductory remarks about the origin of the identities, our paper is entirely combinatorial and does not require any knowledge of Shimura varieties or of representation theory.
\end{abstract}

\section{INTRODUCTION}

The goal of this paper is to give more natural and geometric proofs of some combinatorial identities that appear when one calculates the commuting actions of the Hecke algebra and the absolute Galois group of $\mathbb{Q}$ on the cohomology of a Siegel modular variety. To be more precise, the cohomology that is used is the intersection cohomology of the minimal compactification. These identities, which appear in the calculation of weighted orbital integrals at the real place, are the technical heart of the paper [8], but were relegated to an appendix and proved by brute force.

For the expert, we next provide some details about the manner our combinatorial identities appear in [8]. The calculation in Proposition 3.3.1 of [8], which is the central technical result of that paper, requires us to identify two virtual representations of the real points of a maximal torus $T$ of the general symplectic group $G$. The first expression, called $L_{M}\left(\gamma_{M}\right)$ in [8, Section 1.2], comes from a geometric calculation using the Lefschetz fixed point formula, and involves the action of $T$ on truncated cohomology groups of the Lie algebra of the unipotent radical of parabolic subgroups containing $T$. The second expression, which comes from Arthur and Kottwitz's expression for the spectral side of the stable trace formula, involves averaged characters of discrete series representations of $G(\mathbb{R})$; see [8, Section 3.1]. Both expressions can

Manuscript received 24th June 2018, revised and accepted 18th February 2019.

KEYWORDS. Averaged discrete series characters, permutahedron, intersection cohomology, ordered set partitions, shellability.

ACKNowledgements. This work was partially supported by grants from the Simons Foundation (\#429370 to Richard Ehrenborg and \#422467 to Margaret Readdy). This work was also partially supported by the LABEX MILYON (ANR-10-LABX-0070) of Université de Lyon, within the program "Investissements d'Avenir" (ANR-11-IDEX- 0007) operated by the French National Research Agency (ANR). 
be reformulated as linear combinations of quasi-characters of $T(\mathbb{R})$, the first via formulas of Kostant for the Lie algebra cohomology and Weyl for the character of an algebraic representation, and the second via Harish-Chandra's formula for discrete series characters, made more explicit by Herb in [7]. This is explained in the proof of Proposition 3.3.1 of [8]. When we equate the coefficients of a fixed quasi-character on both sides, we arrive at Corollary A.5 of [8], which follows easily from Proposition A.4 of that article. The proof of that proposition in [8] is long, technical and not exactly enlightening.

In this paper, we present a geometric proof of this result (our Theorem 6.4) which involves the geometry of the Coxeter complex of the symmetric group $\mathfrak{S}_{n}$. This complex can also be described as the boundary of the dual polytope of the permutahedron. This geometric viewpoint also yields a strengthening of Corollary A.3 of [8]. We emphasize that our approach is completely combinatorial. Although our identities originally stem from the representation theory and arithmetic geometry used in the calculation of the cohomology of Shimura varieties, the current paper is directed to a combinatorial audience.

An overview of the paper is as follows. Section 2 contains preliminaries about the permutahedron and shellings. Section 3 gives the definition of the weighted subcomplex $\Sigma(\lambda)$ of the Coxeter complex $\Sigma_{n}$ of the symmetric group $\mathfrak{S}_{n}$ that we wish to study. We prove $\Sigma(\lambda)$ is a pure subcomplex of the same dimension as $\Sigma_{n}$; see Lemma 3.6. In Section 4 we give a brief proof of the theorem (originally due to Björner; see [2]) that any linear extension of the weak Bruhat order is a shelling order on the facets of $\Sigma_{n}$, and deduce a similar result for $\Sigma(\lambda)$. Section 5 yields another proof that the weighted complex $\Sigma(\lambda)$ is shellable by viewing it as the order complex of an $E L$-shellable poset. The shelling results of Sections 4 and 5 imply that $\Sigma(\lambda)$ is always homeomorphic to a ball or a sphere, and imply Corollary A.3 of [8], but they are much stronger than this corollary. In Section 6 we state our main result, Theorem 6.4, which corresponds to Proposition A. 4 of [8]. Sections 7 and 8 contain the proof of this theorem: in Section 7 we give the proof in the case $\lambda$ is weakly increasing, and in Section 8 we show how to reduce the general case to this base case. Finally, in Section 9 we derive another expression for the left-hand side of the identity of Theorem 6.4 when $\lambda$ is a weakly decreasing sequence.

\section{Preliminaries}

2.1. Permutations And ORdered PARtitions. Let $\mathfrak{S}_{n}$ denote the symmetric group on $n$ elements. We write permutations $\tau \in \mathfrak{S}_{n}$ in one-line notation, that is, $\tau=\tau_{1} \tau_{2} \cdots \tau_{n}$. Let the symmetric group $\mathfrak{S}_{n}$ act upon the vector space $\mathbb{R}^{n}$ by permuting the coordinates, that is, given a permutation $\tau$ and the vector $x=\left(x_{1}, x_{2}, \ldots, x_{n}\right)$, we define $\tau(x)=\left(x_{\tau^{-1}(1)}, x_{\tau^{-1}(2)}, \ldots, x_{\tau^{-1}(n)}\right)$. This is a left action, since for two permutations $\tau$ and $\pi$ we have that $\tau(\pi(x))=(\tau \circ \pi)(x)$.

Define $[n]$ to be the set $\{1,2, \ldots, n\}$. We will need the following permutation statistics. The descent set of a permutation $\tau \in \mathfrak{S}_{n}$ is the set $\operatorname{Des}(\tau)=\{i \in[n-1]$ : $\left.\tau_{i}>\tau_{i+1}\right\}$. The descent composition is the list $\left(d_{1}-d_{0}, d_{2}-d_{1}, \ldots, d_{k+1}-d_{k}\right)$ where $\operatorname{Des}(\tau)=\left\{d_{1}<d_{2}<\cdots<d_{k}\right\}$ and we tacitly assume $d_{0}=0$ and $d_{k+1}=n$. The number of inversions of a permutation, also called the length, is given by $\operatorname{inv}(\tau)=$ $\left|\left\{(i, j): 1 \leqslant i<j \leqslant n, \tau_{i}>\tau_{j}\right\}\right|$. Finally, denote the sign of a permutation $\tau$ by $(-1)^{\tau}$, that is, $(-1)^{\tau}=(-1)^{\operatorname{inv}(\tau)}$.

In the symmetric group $\mathfrak{S}_{n}$ let $s_{i}$ denote the simple transposition $(i, i+1)$ where $1 \leqslant i \leqslant n-1$. The weak Bruhat order on the symmetric group $\mathfrak{S}_{n}$ is defined by the 
cover relation $\tau \prec \tau s_{i}$ where $\operatorname{inv}(\tau)<\operatorname{inv}\left(\tau s_{i}\right)$. More explicitly, the cover relation is

$$
\tau_{1} \cdots \tau_{i} \tau_{i+1} \cdots \tau_{n} \prec \tau_{1} \cdots \tau_{i+1} \tau_{i} \cdots \tau_{n} \quad \text { if } \quad \tau_{i}<\tau_{i+1} .
$$

With respect to this partial order, the identity element $12 \cdots n$ is the minimal element, the permutation $n \cdots 21$ is the maximal element, and the rank function is given by the number of inversions of the permutation.

The $(n-1)$-dimensional permutahedron is the simple polytope defined by taking the convex hull of the $n$ ! points $\left(\tau_{1}, \tau_{2}, \ldots, \tau_{n}\right) \in \mathbb{R}^{n}$ where $\tau \in \mathfrak{S}_{n}$. These $n$ ! points lie in the hyperplane $\sum_{i=1}^{n} x_{i}=\left(\begin{array}{c}n+1 \\ 2\end{array}\right)$, and one can verify they generate a polytope of dimension $n-1$. Label the vertex $\left(\tau_{1}, \tau_{2}, \ldots, \tau_{n}\right)$ by the inverse permutation $\tau^{-1}$. The 1-skeleton of the $(n-1)$-dimensional permutahedron yields the Hasse diagram of the weak Bruhat order of $\mathfrak{S}_{n}$, where the cover relations are oriented away from the vertex labeled $12 \cdots n$ and toward the vertex labeled $n \cdots 21$.

A partition $\pi=\left\{B_{1}, B_{2}, \ldots, B_{k}\right\}$ of the set $[n]$ is a collection of subsets of $[n]$, called blocks, such that $B_{i} \neq \varnothing$ for $1 \leqslant i \leqslant k, B_{i} \cap B_{j}=\varnothing$ for $1 \leqslant i<j \leqslant k$ and $\bigcup_{i=1}^{k} B_{i}=[n]$. Let $\Pi_{n}$ denote the collection of all the partitions of the set $[n]$. We make $\Pi_{n}$ into a partially ordered set (poset) by the cover relation

$$
\left\{B_{1}, B_{2}, B_{3}, \ldots, B_{k}\right\} \prec\left\{B_{1} \cup B_{2}, B_{3}, \ldots, B_{k}\right\} .
$$

In other words, $\Pi_{n}$ is ordered by reverse refinement. The poset $\Pi_{n}$ is in fact a lattice, known as the partition lattice. Furthermore, the partition lattice $\Pi_{n}$ is a graded poset, with minimal element $\hat{0}=\{\{1\},\{2\}, \ldots,\{n\}\}$, maximal element $\hat{1}=\{[n]\}$ and rank function $\rho(\pi)=n-|\pi|$, where $|\pi|$ denotes the number of blocks of the partition $\pi$.

An ordered partition $\sigma=\left(C_{1}, C_{2}, \ldots, C_{k}\right)$ of the set $[n]$ is a list of subsets of $[n]$ such that $\left\{C_{1}, C_{2}, \ldots, C_{k}\right\}$ is a partition of $[n]$. Let $\Pi_{n}^{\text {ord }}$ denote the set of all ordered partitions on the set $[n]$. The set $\Pi_{n}^{\text {ord }}$ forms a poset by letting the cover relation be the merging of two adjacent blocks, that is,

$$
\left(C_{1}, \ldots, C_{i}, C_{i+1}, \ldots, C_{k}\right) \prec\left(C_{1}, \ldots, C_{i} \cup C_{i+1}, \ldots, C_{k}\right) .
$$

Observe that the maximal element of $\Pi_{n}^{\text {ord }}$ is the ordered partition consisting of one block $([n])$. However there are $n$ ! minimal elements, one for each permutation $\tau=$ $\tau_{1} \tau_{2} \cdots \tau_{n}$ in the symmetric group $\mathfrak{S}_{n}$, namely the ordered partitions of the form $\left(\left\{\tau_{1}\right\},\left\{\tau_{2}\right\}, \ldots,\left\{\tau_{n}\right\}\right)$. We identify these minimal elements with permutations in $\mathfrak{S}_{n}$ written in one-line notation. Let $|\sigma|$ denote the number of blocks of the ordered partition $\sigma$. Also observe that every interval in $\Pi_{n}^{\text {ord }}$ is isomorphic to a Boolean algebra, that is, the interval $\left[\sigma_{1}, \sigma_{2}\right]$ is isomorphic to the Boolean algebra $B_{\left|\sigma_{1}\right|-\left|\sigma_{2}\right|}$. For recent work regarding ordered partitions, see $[4,5]$.

When we adjoin a minimal element $\hat{0}$ to $\Pi_{n}^{\text {ord }}$ the resulting poset is a lattice, called the ordered partition lattice. In fact, it is the face lattice of the $(n-1)$-dimensional permutahedron.

A composition of $n$ is a list $\left(c_{1}, c_{2}, \ldots, c_{k}\right)$ of positive integers such that $\sum_{i=1}^{k} c_{i}=n$. Let $\operatorname{Comp}(n)$ denote the set of all compositions of $n$. The set $\operatorname{Comp}(n)$ forms a poset by letting the cover relation be the adding of two adjacent entries, that is,

$$
\left(c_{1}, \ldots, c_{i}, c_{i+1}, \ldots, c_{k}\right) \prec\left(c_{1}, \ldots, c_{i}+c_{i+1}, \ldots, c_{k}\right) .
$$

The poset $\operatorname{Comp}(n)$ is isomorphic to the Boolean algebra $B_{n-1}$. Define the map type : $\Pi_{n}^{\text {ord }} \longrightarrow \operatorname{Comp}(n)$ by reading off the cardinalities of the blocks, that is,

$$
\operatorname{type}\left(\left(C_{1}, C_{2}, \ldots, C_{k}\right)\right)=\left(\left|C_{1}\right|,\left|C_{2}\right|, \ldots,\left|C_{k}\right|\right) .
$$

This is an order-preserving map. 
2.2. Shellable and Decomposable simplicial Complexes. For a face $F$ of a simplicial complex $\Delta$ let $\bar{F}$ denote the subcomplex $\{G: G \subseteq F\}$. Recall that a pure simplicial complex $\Delta$ of dimension $d$ is said to be shellable if it is either 0 dimensional, or there is an ordering of the facets $F_{1}, F_{2}, \ldots, F_{s}$ such that the complex $\overline{F_{j}} \cap\left(\bigcup_{i=1}^{j-1} \overline{F_{i}}\right)$ is a pure simplicial complex of dimension $d-1$ for $2 \leqslant j \leqslant s$. As a remark, there are more general formulations of shellability for non-pure complexes and polytopal complexes; see [3]. For a facet $F_{j}$ let $R\left(F_{j}\right)$ be the facet restriction, that is, the smallest dimensional face of $F_{j}$ that does not appear in the complex $\bigcup_{i=1}^{j-1} \overline{F_{i}}$. The shelling condition implies that the face poset of $\Delta$ can be written as the disjoint union $\bigcup_{j=1}^{s}\left[R\left(F_{j}\right), F_{j}\right]$. We call the facet $F_{j}$ a homology facet if $R\left(F_{j}\right)=F_{j}$. It is only for homology facets that the topology of the complex changes in the $j$ th shelling step from $\overline{F_{1}} \cup \cdots \cup \overline{F_{j-1}}$ to $\overline{F_{1}} \cup \cdots \cup \overline{F_{j}}$. The subcomplex $\overline{F_{1}} \cup \cdots \cup \overline{F_{j}}$ is said to be a partial shelling of the complex $\Delta$. See $[1,9]$ and its references for background on shellability.

Recall that a pure simplicial complex $\Delta$ is decomposable if every facet $F_{j}$ has a face $R\left(F_{j}\right)$ such that we can write $\Delta$ as a disjoint union

$$
\Delta=\bigcup_{j=1}^{s}\left[R\left(F_{j}\right), F_{j}\right]
$$

Complexes satisfying this property are usually called partitionable (see [9]), but we use the term "decomposable" here to avoid any confusion with ordered partitions.

A shellable complex is decomposable, but the converse is not true in general. However, the following lemma, which follows easily from Proposition 2.5 of [3], yields a condition which implies shellability.

LeMma 2.1. Let $\Delta$ be a decomposable simplicial complex with the decomposition given by (1). The ordering of the facets $F_{1}, F_{2}, \ldots, F_{s}$ is a shelling order if and only if for every face $G$ of the facet $F_{j}$ there exist an index $i \leqslant j$ such that the face $G$ belongs to the interval $\left[R\left(F_{i}\right), F_{i}\right]$.

For further background on combinatorial structures and posets, see [10]. For more on the combinatorics of simplicial complexes, see [9].

\section{The Weighted COMPlex $\Sigma(\lambda)$}

Let $\lambda$ be a sequence of $n$ real numbers, that is, $\lambda=\left(\lambda_{1}, \lambda_{2}, \ldots, \lambda_{n}\right) \in \mathbb{R}^{n}$. For a subset $S \subseteq[n]$ we introduce the shorthand notation $\lambda_{S}=\sum_{i \in S} \lambda_{i}$. Define the subset $\mathcal{P}(\lambda)$ of the set of ordered partitions $\Pi_{n}^{\text {ord }}$ by

$$
\mathcal{P}(\lambda)=\left\{\sigma=\left(C_{1}, C_{2}, \ldots, C_{k}\right) \in \Pi_{n}^{\text {ord }}: \sum_{i=1}^{j} \lambda_{C_{i}}>0 \text { for } 1 \leqslant j \leqslant k\right\} .
$$

Note that if $\lambda_{1}+\lambda_{2}+\cdots+\lambda_{n} \leqslant 0$ then the set $\mathcal{P}(\lambda)$ is empty.

LEMMA 3.1. The set $\mathcal{P}(\lambda)$ is an upper order ideal (also known as a filter) in the poset $\Pi_{n}^{\text {ord }}$.

This follows directly from the definitions since by merging two adjacent blocks there is one less inequality to verify.

LEMMA 3.2. Given an ordered partition $\sigma=\left(C_{1}, C_{2}, \ldots, C_{k}\right) \in \mathcal{P}(\lambda)$, assume that $C_{j}$ is a non-singleton block. Let a be an element of the block $C_{j}$ with maximal $\lambda$-value, that is, $\lambda_{a}=\max _{b \in C_{j}}\left(\lambda_{b}\right)$. Let $\sigma^{\prime}$ be the ordered partition

$$
\sigma^{\prime}=\left(C_{1}, C_{2}, \ldots, C_{j-1},\{a\}, C_{j}-\{a\}, C_{j+1}, \ldots, C_{k}\right) .
$$

Then the cover relation $\sigma^{\prime} \prec \sigma$ holds. Furthermore, $\sigma^{\prime}$ belongs to the set $\mathcal{P}(\lambda)$. 
Proof. The cover relation $\sigma^{\prime} \prec \sigma$ is immediate. To verify that $\sigma^{\prime} \in \mathcal{P}(\lambda)$ it is enough to verify that $\sum_{i=1}^{j-1} \lambda_{C_{i}}+\lambda_{a}>0$. If $\lambda_{a} \geqslant 0$ this is immediately true. If $\lambda_{a}<0$ then the inequality follows by noticing that all the $\lambda$-values associated to the elements in the block $C_{j}$ are negative and we have that $\sum_{i=1}^{j-1} \lambda_{C_{i}}+\lambda_{a} \geqslant \sum_{i=1}^{j-1} \lambda_{C_{i}}+\lambda_{C_{j}}>0$.

Similar to the previous lemma we have the next result. This lemma will be used in the proof of the main result in Section 8.

LEMMA 3.3. Given an ordered partition $\sigma=\left(C_{1}, C_{2}, \ldots, C_{k}\right) \in \mathcal{P}(\lambda)$, assume that $B$ is a non-empty proper subset of $C_{j}$, that is, $\varnothing \subsetneq B \subsetneq C_{j}$. Let $\sigma^{\prime}$ be the ordered partition

$$
\sigma^{\prime}= \begin{cases}\left(C_{1}, C_{2}, \ldots, C_{j-1}, B, C_{j}-B, C_{j+1}, \ldots, C_{k}\right) & \text { if } \lambda_{B}>0, \\ \left(C_{1}, C_{2}, \ldots, C_{j-1}, C_{j}-B, B, C_{j+1}, \ldots, C_{k}\right) & \text { if } \lambda_{B} \leqslant 0 .\end{cases}
$$

Then the cover relation $\sigma^{\prime} \prec \sigma$ holds. Furthermore, $\sigma^{\prime}$ belongs to the set $\mathcal{P}(\lambda)$.

Proof. Again, the cover relation is immediate. The fact that $\sigma^{\prime} \in \mathcal{P}(\lambda)$ follows by two cases. In the case $\lambda_{B}>0$ it is enough to observe that $\sum_{i=1}^{j-1} \lambda_{C_{i}}+\lambda_{B}>0$. In the second case, we have $\sum_{i=1}^{j-1} \lambda_{C_{i}}+\lambda_{C_{j}-B}=\sum_{i=1}^{j} \lambda_{C_{i}}-\lambda_{B}>0$.

Let $\mathcal{A}(\lambda)$ denote the subset of the symmetric group $\mathfrak{S}_{n}$ defined by

$$
\mathcal{A}(\lambda)=\left\{\tau \in \mathfrak{S}_{n}: \sum_{i=1}^{j} \lambda_{\tau(i)}>0 \text { for } 1 \leqslant j \leqslant n\right\} .
$$

Since permutations correspond to ordered partitions having all singleton blocks, we observe that the set $\mathcal{A}(\lambda)$ is a subset of $\mathcal{P}(\lambda)$. In fact, it is the subset of minimal elements of $\mathcal{P}(\lambda)$.

Lemma 3.4. Let $\lambda \in \mathbb{R}^{n}$ be a sequence such that $\lambda_{1}+\lambda_{2}+\cdots+\lambda_{n}>0$. Then the set $\mathcal{A}(\lambda)$ is nonempty. Especially, any permutation $\tau$ satisfying $\lambda_{\tau_{1}} \geqslant \lambda_{\tau_{2}} \geqslant \cdots \geqslant \lambda_{\tau_{n}}$ belongs to $\mathcal{A}(\lambda)$.

Proof. Since the sum $\sum_{i=1}^{n} \lambda_{i}$ is positive, the ordered partition $([n])$ belongs to $\mathcal{P}(\lambda)$. Now iterating Lemma 3.2 we obtain that the ordered partition corresponding to the permutation $\tau$ belongs to $\mathcal{P}(\lambda)$.

LEMma 3.5. The upper order ideal $\mathcal{P}(\lambda)$ is generated by the set $\mathcal{A}(\lambda)$, that is, for every ordered partition $\sigma \in \mathcal{P}(\lambda)$ there is a permutation $\tau \in \mathcal{A}(\lambda)$ such that $\tau \leqslant \sigma$ in $\Pi_{n}^{\text {ord }}$.

Proof. Begin with the ordered partition $\sigma$ and iterate Lemma 3.2. This procedure yields the permutation $\tau$.

Recall that $\mathcal{P}(\lambda)$ is an upper order ideal in the poset $\Pi_{n}^{\text {ord }}$, and that $\Pi_{n}^{\text {ord }}$ is a joinsemilattice where each interval is isomorphic to a Boolean algebra. Hence by reversing the order relations of $\Pi_{n}^{\text {ord }}$ we obtain the face poset of a simplicial complex, and we can view the set $\mathcal{P}(\lambda)$ as a simplicial subcomplex $\Sigma(\lambda)$. We call $\Sigma(\lambda)$ the weighted complex. See Figure 1 for an example. The maximal element $\hat{1}$ of $\mathcal{P}(\lambda)$ is the empty face of the simplicial complex $\Sigma(\lambda)$ and the minimal elements $\mathcal{A}(\lambda)$ are the facets of $\Sigma(\lambda)$. Note that if $\lambda_{1}+\lambda_{2}+\cdots+\lambda_{n} \leqslant 0$ then $\Sigma(\lambda)$ is the empty simplicial complex, which has no faces, not even the empty face.

This idea of turning an upper order ideal upside-down in order to view it as a simplicial complex appears in $[4,5]$.

Lemma 3.5 can now be reformulated as follows.

Lemma 3.6. Let $\lambda \in \mathbb{R}^{n}$ be a sequence such that $\lambda_{1}+\lambda_{2}+\cdots+\lambda_{n}>0$. Then the weighted complex $\Sigma(\lambda)$ is a pure simplicial complex of dimension $n-2$. 




Figure 1. The simplicial complex $\Sigma(\lambda)$ for $\lambda=(5,1,-2,-3)$ consisting of 7 vertices, 12 edges and 6 triangles. The empty face, labeled 1234, is not depicted.

Reordering the entries of the sequence $\lambda$ does not change the complex as the following lemma shows.

Lemma 3.7. If $\tau \in \mathfrak{S}_{n}$, then the complexes $\Sigma(\lambda)$ and $\Sigma(\tau(\lambda))$ are isomorphic under the bijection $\Sigma(\lambda) \longrightarrow \Sigma(\tau(\lambda))$ defined by $\sigma \longmapsto \tau(\sigma)$.

Proof. It is enough to observe that $\tau(\lambda)_{\tau(B)}=\lambda_{B}$ for all subsets $B \subseteq[n]$.

\section{Shellings of the Coxeter COMPlex}

Let $\Sigma_{n}$ be the simplicial complex formed by the boundary of the dual of the $(n-1)$ dimensional permutahedron. This is the type $A$ Coxeter complex. The face poset of $\Sigma_{n}$ is the dual poset $\left(\Pi_{n}^{\text {ord }}\right)^{*}$ with the order relation of the faces in $\Sigma_{n}$ by $\leqslant^{*}$, the dual order. The permutations in $\mathfrak{S}_{n}$ correspond to the facets of $\Sigma_{n}$. We begin by showing that $\Sigma_{n}$ is a decomposable complex.

Recall that the descent set for a permutation $\tau \in \mathfrak{S}_{n}$ is the set $\operatorname{Des}(\tau)=\left\{d_{1}<\right.$ $\left.d_{2}<\cdots<d_{k}\right\}$ such that

$$
\begin{aligned}
& \tau_{1}<\tau_{2}<\cdots<\tau_{d_{1}}>\tau_{d_{1}+1}<\tau_{d_{1}+2}<\cdots<\tau_{d_{2}}>\tau_{d_{2}+1}<\cdots<\tau_{d_{k}} \\
& >\tau_{d_{k}+1}<\tau_{d_{k}+2}<\cdots<\tau_{n} .
\end{aligned}
$$

Define the ordered partition $R(\tau)$ to be

$$
R(\tau)=\left(\left\{\tau_{1}, \tau_{2}, \ldots, \tau_{d_{1}}\right\},\left\{\tau_{d_{1}+1}, \tau_{d_{1}+2}, \ldots, \tau_{d_{2}}\right\}, \ldots,\left\{\tau_{d_{k}+1}, \tau_{d_{k}+2}, \ldots, \tau_{n}\right\}\right) .
$$

Note that the blocks of $R(\tau)$ consist of the maximal ascending runs in the permutation $\tau$.

Define the map $f: \Sigma_{n} \longrightarrow \mathfrak{S}_{n}$ by taking an ordered partition, ordering the elements in each block in increasing order and then recording the elements as a permutation by 
reading from left to right. Observe that, for every ordered partition $\sigma$, the permutation $f(\sigma)$ is the minimal permutation in the weak Bruhat order such that $\sigma \leqslant{ }^{*} f(\sigma)$, and it is also the permutation of minimal length such that $\sigma \leqslant{ }^{*} f(\sigma)$.

The next two results are due to Björner; see [2, Theorem 2.1].

Proposition 4.1 (Björner). The simplicial complex $\Sigma_{n}$ is decomposable, that is, it is given by the disjoint union

$$
\Sigma_{n}=\bigcup_{\tau \in \mathfrak{S}_{n}}[R(\tau), \tau]
$$

Proof. Define the map $r: \Sigma_{n} \longrightarrow \Sigma_{n}$ by iterating the following procedure. If $C_{i}$ and $C_{i+1}$ are two adjacent blocks in the ordered partition $\sigma$ such that $\max \left(C_{i}\right)<$ $\min \left(C_{i+1}\right)$ then merge these two blocks together. It is clear that we have the inequalities $r(\sigma) \leqslant^{*} \sigma \leqslant^{*} f(\sigma)$. Also we have that $f(r(\sigma))=f(\sigma)$ and $r(f(\sigma))=r(\sigma)$. Furthermore for a permutation (facet) $\tau$ we have that $R(\tau)=r(\tau)$. Hence each ordered partition $\sigma$ appears only in the interval $[r(f(\sigma)), f(\sigma)]$. Thus each ordered partition $\sigma$ appears exactly once in the union (2), and hence this union is disjoint.

THEOREM 4.2 (Björner). Any linear extension of the weak Bruhat order is a shelling order of the simplicial complex $\Sigma_{n}$.

Proof. Fix a linear extension of the weak Bruhat order. Denote it by $<_{e}$. Consider a permutation $\tau \in \mathfrak{S}_{n}$. Let $\sigma$ be an ordered partition such that $\sigma \leqslant{ }^{*} \tau$, that is, $\sigma$ is a face of the facet $\tau$. Now consider the permutation $f(\sigma)$. Note that the permutation $f(\sigma)$ is obtained from the permutation $\tau$ by merging elements together to form blocks and then sorting the elements in each block in increasing order. Hence in the weak Bruhat order we have $f(\sigma) \leqslant \tau$. Hence $f(\sigma)$ appears earlier in the linear extension than $\tau$, that is, $f(\sigma) \leqslant_{e} \tau$. The result follows by Lemma 2.1 .

We now consider our complex $\Sigma(\lambda)$.

THEOREM 4.3. Let $\lambda=\left(\lambda_{1}, \ldots, \lambda_{n}\right)$ be a sequence of $n$ real numbers such that $\lambda_{1}+$ $\lambda_{2}+\cdots+\lambda_{n}>0$. Then the complex $\Sigma(\lambda)$ is shellable. Furthermore, the complex $\Sigma(\lambda)$ is homeomorphic to a sphere or a ball according to

$$
\Sigma(\lambda) \cong \begin{cases}\mathbb{S}^{n-2} & \text { if } \lambda_{1}, \lambda_{2}, \ldots, \lambda_{n}>0 \\ \mathbb{B}^{n-2} & \text { otherwise. }\end{cases}
$$

The proof of Theorem 4.3 will appear directly after the proof of Proposition 4.7.

Proposition 4.4. Let $\lambda \in \mathbb{R}^{n}$ be a sequence such that $\lambda_{1} \geqslant \lambda_{2} \geqslant \cdots \geqslant \lambda_{n}$ and $\lambda_{1}+\lambda_{2}+\cdots+\lambda_{n}>0$. Then the elements $\mathcal{A}(\lambda)$ form a lower order ideal with respect to the weak Bruhat order on the symmetric group $\mathfrak{S}_{n}$.

Proof. Assume that we have the following cover relation in the weak Bruhat order:

$$
\tau=\tau_{1} \cdots \tau_{j} \tau_{j+1} \cdots \tau_{n} \prec \tau_{1} \cdots \tau_{j+1} \tau_{j} \cdots \tau_{n}=\tau^{\prime},
$$

where $\tau_{j}<\tau_{j+1}$, and suppose that $\tau^{\prime} \in \mathcal{A}(\lambda)$. To verify that $\tau$ belongs to $\mathcal{A}(\lambda)$, it is enough to verify that $\sum_{i=1}^{j} \lambda_{\tau_{i}}$ is nonnegative. Note that $\tau_{j}<\tau_{j+1}$ implies $\lambda_{\tau_{j}} \geqslant \lambda_{\tau_{j+1}}$. So $\sum_{i=1}^{j} \lambda_{\tau_{i}} \geqslant \lambda_{\tau_{j+1}}+\sum_{i=1}^{j-1} \lambda_{\tau_{i}}$ which is positive by our assumption. Hence $\mathcal{A}(\lambda)$ is closed under the cover relation and hence it is a lower order ideal in the weak Bruhat order.

REMARK 4.5. A similar proof also shows that $\mathcal{A}(\lambda)$ is a lower order ideal with respect to the strong Bruhat order. 
Proposition 4.6. Let $\lambda \in \mathbb{R}^{n}$ be a sequence such that $\lambda_{1} \geqslant \lambda_{2} \geqslant \cdots \geqslant \lambda_{n}$. Then the weighted complex $\Sigma(\lambda)$ has the decomposition

$$
\Sigma(\lambda)=\bigcup_{\tau \in \mathcal{A}(\lambda)}[R(\tau), \tau]
$$

Proof. By Proposition 4.4 the intersection $\Sigma(\lambda) \cap[R(\tau), \tau]$ is empty if $\tau$ is not in $\mathcal{A}(\lambda)$ and otherwise is the entire interval $[R(\tau), \tau]$. Hence the result follows from Proposition 4.1.

Proposition 4.7. Let $\lambda \in \mathbb{R}^{n}$ be a sequence such that $\lambda_{1} \geqslant \lambda_{2} \geqslant \cdots \geqslant \lambda_{n}$ and $\lambda_{1}+\lambda_{2}+\cdots+\lambda_{n}>0$. Then the weighted complex $\Sigma(\lambda)$ is a partial shelling of $\Sigma_{n}$ and hence $\Sigma(\lambda)$ is shellable. Furthermore, the complex $\Sigma(\lambda)$ is homeomorphic to a sphere or a ball according to

$$
\Sigma(\lambda) \cong \begin{cases}\mathbb{S}^{n-2} & \text { if } \lambda_{n}>0 \\ \mathbb{B}^{n-2} & \text { if } \lambda_{n} \leqslant 0 .\end{cases}
$$

Proof. The first statement follows by combining Theorem 4.2 and Propositions 4.4 and 4.6. The second statement follows from the fact that $\Sigma_{n}$ is a sphere. The only homology facet of the complex $\Sigma_{n}$ is the permutation $n \cdots 21$ and it only appears as a facet in $\Sigma(\lambda)$ if $\lambda_{n}>0$.

The proof of Theorem 4.3 now follows directly by combining Lemma 3.7 and Proposition 4.7. By taking the reduced Euler characteristic of the simplicial complex $\Sigma(\lambda)$ in Theorem 4.3, we obtain the following result that appears in [8, Corollary A.3] :

Corollary 4.8. Let $\lambda \in \mathbb{R}^{n}$. Then

$$
\sum_{\sigma \in \mathcal{P}(\lambda)}(-1)^{|\sigma|}= \begin{cases}(-1)^{n} & \text { if } \lambda_{1}, \lambda_{2}, \ldots, \lambda_{n}>0 \\ 0 & \text { otherwise. }\end{cases}
$$

This corollary can also be proven by a sign-reversing involution on the set $\mathcal{P}(\lambda)$. Furthermore, such a sign-reversing involution could also be made into a discrete Morse matching; see [6]. However, the discrete Morse matching would only give the weaker topologicial result that $\Sigma(\lambda)$ is either contractible when $\lambda_{i} \leqslant 0$ for some index $i$ or homotopy equivalent to a sphere when $\lambda_{1}, \lambda_{2}, \ldots, \lambda_{n}>0$.

\section{The LEXICOGRAPHIC SHELLING}

In this section we give a second proof that the weighted complex $\Sigma(\lambda)$ is shellable by viewing it as the order complex of an $E L$-shellable poset. Throughout in this section we assume that the entries of $\lambda$ are pairwise distinct. We can always perturb $\lambda$ slightly to make this condition true. This does not change the complex $\Sigma(\lambda)$. We also assume that $\lambda_{1}+\lambda_{2}+\cdots+\lambda_{n}>0$.

Let $P$ be a graded poset that has a minimal element $\hat{0}$ and a maximal element $\hat{1}$ and where all maximal chains have the same length. Let $\mathcal{C}(P)$ be the set of all cover relations of $P$, that is,

$$
\mathcal{C}(P)=\left\{(x, y) \in P^{2} \quad: \quad x \prec y\right\} .
$$

A labeling of a poset $P$ is a map $\kappa$ from the set of cover relations $\mathcal{C}(P)$ to a linearly ordered set. For a saturated chain $c=\left\{x_{0} \prec x_{1} \prec \cdots \prec x_{k}\right\}$ let $\kappa(c)$ denote the word of labels, that is, $\kappa(c)=\kappa\left(x_{0}, x_{1}\right) \kappa\left(x_{1}, x_{2}\right) \cdots \kappa\left(x_{k-1}, x_{k}\right)$. For a graded poset $P$, an $R$ labeling is a labeling $\kappa$ such that in each interval $[x, y]$ there is a unique chain $c=\{x=$ $x_{0} \prec x_{1} \prec \cdots \prec x_{k}=y$ \} where the word $\kappa(c)$ is rising, that is, $\kappa\left(x_{0}, x_{1}\right) \leqslant \kappa\left(x_{1}, x_{2}\right) \leqslant$ 
$\cdots \leqslant \kappa\left(x_{k-1}, x_{k}\right)$. An EL-labeling is an $R$-labeling with the extra condition that the word $\kappa(c)$ corresponding to the unique rising chain $c$ in the interval $[x, y]$ is also lexicographically least among the set of all the words arising from saturated chains in the interval. It is well-known that a poset having an $E L$-labeling implies that the order complex $\Delta(P-\{\hat{0}, \hat{1}\})$ is a shellable simplicial complex; see [1, Section 2].

Let $B_{n}$ denote the Boolean algebra, that is, the collection of subsets of the set $[n]$ ordered by inclusion. Define the subposet $B(\lambda)$ by

$$
B(\lambda)=\left\{S \in B_{n} \quad: \lambda_{S}>0\right\} \cup\{\varnothing\} .
$$

Note that the full set $[n]$ belongs to $B(\lambda)$ and it is the maximal element of $B(\lambda)$.

We view this definition geometrically as follows. Identify the vertices of the $n$ dimensional cube, that is, $\{0,1\}^{n}$ with the Boolean algebra $B_{n}$. The subposet $B(\lambda)$ then consists of those vertices $\left(x_{1}, x_{2}, \ldots, x_{n}\right)$ that satisfy the linear inequality $\lambda_{1} x_{1}+$ $\lambda_{2} x_{2}+\cdots+\lambda_{n} x_{n}>0$ as well as the zero vector.

Lemma 5.1. Let $S$ and $T$ be two subsets in the poset $B(\lambda)$ such that $T \subsetneq S$. Let a be the element in the set difference $S-T$ with the maximal $\lambda$-value. Then the set $T \cup\{a\}$ also belongs to $B(\lambda)$.

Proof. The proof is the same as the proof of Lemma 3.2. If $\lambda_{a} \geqslant 0$ then $\lambda_{T \cup\{a\}}=$ $\lambda_{T}+\lambda_{a}>0$. If $\lambda_{a} \leqslant 0$ then all the elements in $S-T$ have non-positive $\lambda$-values, hence $\lambda_{T \cup\{a\}} \geqslant \lambda_{S}>0$.

From this straightforward observation we have the following consequences.

Proposition 5.2. The poset $B(\lambda)$ is graded of rank $n$. Furthermore, by labeling the cover relation $T \subsetneq S$, where $|T|+1=|S|$, by $-\lambda_{a}$, where $a$ is the unique element in the set difference $S-T$, we obtain an EL-labeling of the poset $B(\lambda)$.

Proof. By repeatedly applying Lemma 5.1 we obtain that every interval $[T, S]$ has a saturated chain consisting of $|S|-|T|$ steps. Thus the poset $B(\lambda)$ has cardinality as a rank function. Observe that the labels of any saturated chain in the interval $[T, S]$ are the negatives of the $\lambda$-values of a permutation of elements in the set difference $S-T$. Also, the chain obtained by repeatedly applying Lemma 5.1 has its labels in increasing order. It is the only such increasing chain. Furthermore, it is also the lexicographically least chain. Hence the labeling is an $E L$-labeling.

Finally observe that the order complex of $B(\lambda)$, that is, $\Delta(B(\lambda)-\{\hat{0}, \hat{1}\})$, is the complex $\Sigma(\lambda)$. Thus we obtain that we can shell the facets of $\Sigma(\lambda)$ in lexicographic order.

\section{The MAIN RESUlt}

Define the map $g: \Sigma(\lambda) \longrightarrow \mathfrak{S}_{n}$ by taking an ordered partition $\sigma$, ordering the elements in each block in decreasing order, and then recording the elements as a permutation by reading from left to right. This is similar to the map $f$ defined before Proposition 4.1. The signs of the two permutations $f(\sigma)$ and $g(\sigma)$ are related by

$$
(-1)^{g(\sigma)}=(-1)^{\sum_{i=1}^{k}\left(\begin{array}{c}
c_{i} \\
2
\end{array}\right)} \cdot(-1)^{f(\sigma)},
$$

where $\sigma=\left(C_{1}, C_{2}, \ldots, C_{k}\right)$ and $c_{i}=\left|C_{i}\right|$.

Define $S(\lambda)$ to be the sum

$$
S(\lambda)=\sum_{\sigma \in \Sigma(\lambda)}(-1)^{|\sigma|} \cdot(-1)^{g(\sigma)} .
$$


Let $M_{n}$ be the set of all maximal matchings on the set $\{1,2, \ldots, n\}$. We say that two edges $\{a, c\}$ and $\{b, d\}$ of a matching $p$ cross if $a<b<c<d$. Let $\operatorname{cross}(p)$ denote the number of crossings. Define the sign of a matching $p$ in $M_{n}$ by

$$
(-1)^{p}=(-1)^{\operatorname{cross}(p)} \cdot \begin{cases}1 & \text { if } n \text { is even } \\ (-1)^{i-1} & \text { if } n \text { is odd }\end{cases}
$$

where $i$ is the unique isolated vertex if $n$ is odd.

REMARK 6.1 . When $n$ is odd there is a sign-preserving bijection between $M_{n}$ and $M_{n+1}$ by joining the isolated vertex $i$ to the new vertex $n+1$.

Define two functions $c_{1}: \mathbb{R} \rightarrow \mathbb{R}$ and $c_{2}: \mathbb{R}^{2} \rightarrow \mathbb{R}$ in the following manner:

$$
c_{1}=\mathbb{1}_{\mathbb{R}_{>0}}, \quad \text { and } \quad c_{2}(a, b)= \begin{cases}1 & \text { if } a, b>0 \\ 2 & \text { if } a>-b \geqslant 0 \\ 0 & \text { otherwise }\end{cases}
$$

If $e=\{i, j\}$ is an edge with $i<j$, we set $c(e, \lambda)=c_{2}\left(\lambda_{i}, \lambda_{j}\right)$.

Let $m$ be the number of edges of a maximal matching, that is, $m=\lfloor n / 2\rfloor$. Let $p$ be a maximal matching in $M_{n}$. If $n$ is even, we write $p$ as the collection of edges $\left\{e_{1}, e_{2}, \ldots, e_{m}\right\}$. If $n$ is odd, the matching $p$ is of the form $\left\{e_{1}, e_{2}, \ldots, e_{m}\right\}$ together with an isolated vertex $i$. We set

$$
c(p, \lambda)=\prod_{j=1}^{m} c\left(e_{j}, \lambda\right) \cdot \begin{cases}1 & \text { if } n \text { is even } \\ c_{1}\left(\lambda_{i}\right) & \text { if } n \text { is odd }\end{cases}
$$

Finally, let

$$
T(\lambda)=\sum_{p \in M_{n}}(-1)^{p} \cdot c(p, \lambda) .
$$

REMARK 6.2. Following [7] let $\mathfrak{S}_{n}^{* *}$ be the set of all permutations $\tau$ in the symmetric group $\mathfrak{S}_{n}$ satisfying the inequalities $\tau_{2 j-1}<\tau_{2 j}$ for $1 \leqslant j \leqslant m$ and $\tau_{1}<\tau_{3}<$ $\cdots<\tau_{2 m-1}$. Then there is a bijection $\mathfrak{S}_{n}^{* *} \stackrel{\sim}{\rightarrow} M_{n}$ by sending $\tau$ to the matching $p=\left\{\left\{\tau_{1}, \tau_{2}\right\},\left\{\tau_{3}, \tau_{4}\right\}, \ldots,\left\{\tau_{2 m-1}, \tau_{2 m}\right\}\right\}$. Note that when $n$ is odd, the isolated vertex is $\tau_{n}$. This bijection preserves the sign, that is, $(-1)^{\tau}=(-1)^{p}$.

REMARK 6.3. When $n$ is even we can express the sum $T(\lambda)$ as the Pfaffian of a skewsymmetric matrix. Let $A$ be the skew-symmetric matrix of order $n$ with the upper triangular entries given by $A_{i, j}=c_{2}\left(\lambda_{i}, \lambda_{j}\right)$. The Pfaffian of $A$ is then the sum $T(\lambda)$. (See Remark 6.2.)

Similarly, when $n$ is odd, we use the bijection in Remark 6.1. In this case let $A$ be the skew-symmetric matrix of order $n+1$ where the upper triangular entries are

$$
A_{i, j}= \begin{cases}c_{2}\left(\lambda_{i}, \lambda_{j}\right) & \text { if } 1 \leqslant i<j \leqslant n \\ c_{1}\left(\lambda_{i}\right) & \text { if } 1 \leqslant i \leqslant n, j=n+1 .\end{cases}
$$

Again the Pfaffian of $A$ is the sum $T(\lambda)$.

We can now state the main result. This is Proposition A.4 of [8].

Theorem 6.4. For every $\lambda \in \mathbb{R}^{n}$, we have

$$
S(\lambda)=(-1)^{n} \cdot T(\lambda) .
$$

The proof of Theorem 6.4 will be given at the end of Section 8 . 


\section{The BASE CASE}

Let $\bar{\lambda}$ denote the reverse of $\lambda$, that is, $\bar{\lambda}=\left(\lambda_{n}, \ldots, \lambda_{2}, \lambda_{1}\right)$.

Lemma 7.1. The sum $S(\lambda)$ can be expressed as

$$
\sum_{\sigma \in \Sigma(\lambda)}(-1)^{|\sigma|} \cdot(-1)^{g(\sigma)}=(-1)^{\left(\begin{array}{c}
n \\
2
\end{array}\right)} \cdot \sum_{\sigma \in \Sigma(\bar{\lambda})}(-1)^{|\sigma|} \cdot(-1)^{f(\sigma)} .
$$

Proof. Let $\tau_{0}$ denote the longest permutation in $\mathfrak{S}_{n}$, that is, $\tau_{0}=n \cdots 21$. Note that $(-1)^{\tau_{0}}=(-1)^{\left(\begin{array}{l}n \\ 2\end{array}\right)}$ and $\bar{\lambda}=\tau_{0} \lambda$. For any ordered partition $\sigma \in \Pi_{n}^{\text {ord }}$ we have the following equality between permutations: $\tau_{0} g(\sigma)=f\left(\tau_{0} \sigma\right)$. In particular, their signs agree, that is, $(-1)^{\left(\begin{array}{l}n \\ 2\end{array}\right)} \cdot(-1)^{g(\sigma)}=(-1)^{f\left(\tau_{0} \sigma\right)}$. Now apply Lemma 3.7 using the permutation $\tau_{0}$. The result follows after summing over all faces $\sigma \in \Sigma(\lambda)$.

Proposition 7.2. Let $\lambda \in \mathbb{R}^{n}$ be a sequence such that $\lambda_{1} \leqslant \lambda_{2} \leqslant \cdots \leqslant \lambda_{n}$. Then the sum $S(\lambda)$ is given by

$$
S(\lambda)= \begin{cases}(-1)^{n} & \text { if } \lambda_{1}>0 \\ 0 & \text { otherwise }\end{cases}
$$

Proof. Lemma 7.1 asserts that

$$
S(\lambda)=(-1)^{\left(\begin{array}{l}
n \\
2
\end{array}\right)} \cdot \sum_{\sigma \in \Sigma(\bar{\lambda})}(-1)^{|\sigma|} \cdot(-1)^{f(\sigma)} .
$$

Moreover, the entries of $\bar{\lambda}$ are in weakly decreasing order. Hence we can use the decomposition in Proposition 4.6 for the complex $\Sigma(\bar{\lambda})$, that is, we have

$$
\Sigma(\bar{\lambda})=\bigcup_{\tau \in \mathcal{A}(\bar{\lambda})}[R(\tau), \tau]
$$

Observe the function $(-1)^{f(\sigma)}$ is constant on the interval $[R(\tau), \tau]$. Hence the sum

$$
\sum_{\sigma \in[R(\tau), \tau]}(-1)^{|\sigma|} \cdot(-1)^{f(\sigma)}
$$

is zero unless $R(\tau)=\tau$. But we can only have $R(\tau)=\tau$ when $\tau$ is the longest permutation $n \cdots 21$. For this permutation the sum is $(-1)^{n} \cdot(-1)^{\left(\begin{array}{l}n \\ 2\end{array}\right)}$. This permutation occurs in $\mathcal{A}(\bar{\lambda})$ if and only if $\lambda_{1}>0$. Hence the result follows.

LEMma 7.3. The following two evaluations of $T(\lambda)$ hold:

(1) If $\lambda_{1}, \lambda_{2}, \ldots, \lambda_{n}>0$, then $T(\lambda)=1$.

(2) If $\lambda_{1} \leqslant 0$, then $T(\lambda)=0$.

Proof. We begin by proving the first statement. By the hypothesis on $\lambda$, for every maximal matching $p$, we have $c(p, \lambda)=1$. We prove $T(\lambda)=1$ for all even $n$ by induction. The induction basis $n=2$ is straightforward to check since there is only one maximal matching on two vertices. Assume now it is true for $n-2$. Observe that the parity of the number of crossings to which the edge $\{1, j\}$ contributes is the same as the parity of $j$. Let $q \in M_{n-2}$ be the matching obtained from $p$ by removing the vertices 1 and $j$, and then applying the unique order-preserving relabeling of the remaining vertices. This gives a bijection from the set of maximal matchings $p \in M_{n}$ 
such that 1 and $j$ are matched and the set of maximal matchings $q \in M_{n-2}$, and we have $(-1)^{p}=(-1)^{j}(-1)^{q}$. Hence the sum is given by

$$
T(\lambda)=\sum_{p \in M_{n}}(-1)^{p}=\sum_{j=2}^{n}(-1)^{j} \cdot \sum_{q \in M_{n-2}}(-1)^{q}=\sum_{j=2}^{n}(-1)^{j}=1,
$$

where the third step is the induction hypothesis.

Assume now that $n$ is odd. By using the sign-preserving bijection in Remark 6.1 between $M_{n}$ and $M_{n+1}$, we obtain that the two sums $\sum_{p \in M_{n}}(-1)^{p}$ and $\sum_{r \in M_{n+1}}(-1)^{r}$ are equal, and we have already seen that the second sum is equal to 1 .

We next prove the second statement. Suppose that $\lambda_{1} \leqslant 0$. Then for every maximal matching $p$, we claim $c(p, \lambda)=0$. Indeed, if the vertex 1 is isolated then $c_{1}\left(\lambda_{1}\right)=0$ is a factor of $c(p, \lambda)$. If not, let $e=\{1, j\}$ be the edge of $p$ containing the vertex 1 . The contribution of $e$ to $c(p, \lambda)$ is $c_{2}\left(\lambda_{1}, \lambda_{j}\right)=0$ which is independent of the value of $\lambda_{j}$. In both cases, $c(p, \lambda)$ has a factor equal to 0 , so it is 0 .

By combining Proposition 7.2 and Lemma 7.3, we obtain the following corollary.

Corollary 7.4. If $\lambda_{1} \leqslant \lambda_{2} \leqslant \cdots \leqslant \lambda_{n}$, then $S(\lambda)=(-1)^{n} \cdot T(\lambda)$.

\section{Permuting the entries of $\lambda$}

We now give a way to reduce the general case to the case treated in the previous section, assuming that we know the identity for smaller values of $n$. This reduction is directly inspired by Herb's paper [7] on discrete series characters.

Let $\lambda \in \mathbb{R}^{n}$ with no ordering hypothesis on $\lambda$. Suppose that $n \geqslant 2$, and fix $1 \leqslant i \leqslant$ $n-1$. Recall that $s_{i}$ is the simple transposition $(i, i+1)$ in the symmetric group $\mathfrak{S}_{n}$. Also, we write $\mu=\left(\lambda_{1}, \ldots, \lambda_{i-1}, \lambda_{i+2}, \ldots, \lambda_{n}\right) \in \mathbb{R}^{n-2}$.

Proposition 8.1. The following two identities hold:

$$
\begin{aligned}
& S(\lambda)+S\left(s_{i} \lambda\right)=-2 \cdot \mathbb{1}_{\lambda_{i}+\lambda_{i+1}>0} \cdot S(\mu), \\
& T(\lambda)+T\left(s_{i} \lambda\right)=2 \cdot \mathbb{1}_{\lambda_{i}+\lambda_{i+1}>0} \cdot T(\mu) .
\end{aligned}
$$

Proof. We begin by proving (5). For $\sigma \in \Sigma_{n}$, let $s_{i} \sigma$ denote the ordered partition where we exchange the elements $i$ and $i+1$. Note that $s_{i}$ is an involution on $\Sigma_{n}$ and that it preserves the number of blocks. We write $\Sigma(\lambda)=\Sigma^{\prime}(\lambda) \sqcup \Sigma^{\prime \prime}(\lambda)$, where $\Sigma^{\prime \prime}(\lambda)$ is the set of fixed points of $s_{i}$ in $\Sigma(\lambda)$, that is, $\Sigma^{\prime}(\lambda)$ is the set of $\sigma \in \Sigma(\lambda)$ such that $i$ and $i+1$ are in different blocks of $\sigma$ and $\Sigma^{\prime \prime}(\lambda)$ is the set of $\sigma \in \Sigma(\lambda)$ such that $i$ and $i+1$ are in the same block of $\sigma$.

Note that the action of $s_{i}$ gives a bijection between $\Sigma^{\prime}(\lambda)$ and $\Sigma^{\prime}\left(s_{i} \lambda\right)$. Furthermore, for an ordered partition $\sigma$ in $\Sigma^{\prime}(\lambda)$ we have $g\left(s_{i} \sigma\right)=s_{i} \cdot g(\sigma)$. We obtain

$$
\sum_{\sigma \in \Sigma^{\prime}(\lambda)}(-1)^{|\sigma|} \cdot(-1)^{g(\sigma)}+\sum_{\sigma \in \Sigma^{\prime}\left(s_{i}(\lambda)\right)}(-1)^{|\sigma|} \cdot(-1)^{g(\sigma)}=0 .
$$

We next consider $\Sigma^{\prime \prime}(\lambda)$. Note that $\Sigma^{\prime \prime}(\lambda)=\Sigma^{\prime \prime}\left(s_{i} \lambda\right)$, so it remains to show that

$$
\sum_{\sigma \in \Sigma^{\prime \prime}(\lambda)}(-1)^{|\sigma|} \cdot(-1)^{g(\sigma)}=-\mathbb{1}_{\lambda_{i}+\lambda_{i+1}>0} \cdot S(\mu) .
$$

Suppose first that $\lambda_{i}+\lambda_{i+1} \leqslant 0$. Define a matching on $\Sigma^{\prime \prime}(\lambda)$ in the following manner. If $\sigma=\left(C_{1}, C_{2}, \ldots, C_{r}\right) \in \Sigma^{\prime \prime}(\lambda)$ satisfies $i, i+1 \in C_{s}$ and $\left|C_{s}\right| \geqslant 3$, match it with

$$
\sigma^{\prime}=\left(C_{1}, \ldots, C_{s-1}, C_{s}-\{i, i+1\},\{i, i+1\}, C_{s+1}, \ldots, C_{r}\right)
$$


The hypothesis that $\lambda_{i}+\lambda_{i+1} \leqslant 0$ and Lemma 3.3 imply that $\sigma^{\prime} \in \Sigma^{\prime \prime}(\lambda)$. Furthermore, this is a perfect matching since no ordered partition in $\Sigma^{\prime \prime}(\lambda)$ can begin with the block $\{i, i+1\}$. It is easy to see that $(-1)^{g(\sigma)}=(-1)^{g\left(\sigma^{\prime}\right)}$ if $\sigma$ and $\sigma^{\prime}$ are matched. So we obtain

$$
\sum_{\sigma \in \Sigma^{\prime \prime}(\lambda)}(-1)^{|\sigma|} \cdot(-1)^{g(\sigma)}=0 .
$$

Now assume that $\lambda_{i}+\lambda_{i+1}>0$. We consider another matching on $\Sigma^{\prime \prime}(\lambda)$, defined as follows. If $\sigma=\left(C_{1}, C_{2}, \ldots, C_{r}\right) \in \Sigma^{\prime \prime}(\lambda)$ satisfies $i, i+1 \in C_{s}$ and $\left|C_{s}\right| \geqslant 3$, match it with

$$
\sigma^{\prime}=\left(C_{1}, \ldots, C_{s-1},\{i, i+1\}, C_{s}-\{i, i+1\}, C_{s+1}, \ldots, C_{r}\right) .
$$

The hypothesis that $\lambda_{i}+\lambda_{i+1}>0$ and Lemma 3.3 imply that $\sigma^{\prime} \in \Sigma^{\prime \prime}(\lambda)$. The unmatched elements are the ordered partitions whose last block is $\{i, i+1\}$. Again, it is straightforward to see that $(-1)^{g(\sigma)}=(-1)^{g\left(\sigma^{\prime}\right)}$ if $\sigma$ and $\sigma^{\prime}$ are matched. Denote by $\Sigma^{\prime \prime \prime}(\lambda)$ the set of unmatched elements in $\Sigma^{\prime \prime}(\lambda)$. We obtain

$$
\sum_{\sigma \in \Sigma^{\prime \prime}(\lambda)}(-1)^{|\sigma|} \cdot(-1)^{g(\sigma)}=\sum_{\sigma \in \Sigma^{\prime \prime \prime}(\lambda)}(-1)^{|\sigma|} \cdot(-1)^{g(\sigma)} .
$$

Identify $[n-2]$ and $[n]-\{i, i+1\}$ using the unique order-preserving bijection between these sets. We note that $\sigma=\left(C_{1}, \ldots, C_{r},\{i, i+1\}\right) \in \Sigma_{n}$ is an element of $\Sigma(\lambda)$ if and only if $\tau:=\left(C_{1}, \ldots, C_{r}\right)$ is an element of $\Sigma(\mu)$. This induces a bijection $\Sigma^{\prime \prime \prime}(\lambda) \stackrel{\sim}{\rightarrow}$ $\Sigma(\mu)$. Also, we have $|\sigma|=|\tau|+1$ and $(-1)^{g(\sigma)}=(-1)^{g(\tau)}$. So finally we find that

$$
\sum_{\sigma \in \Sigma^{\prime \prime \prime}(\lambda)}(-1)^{|\sigma|} \cdot(-1)^{g(\sigma)}=-\sum_{\tau \in \Sigma(\mu)}(-1)^{|\tau|} \cdot(-1)^{g(\tau)}=-S(\mu) .
$$

Identity (5) follows by combining equation (7) with twice equation (8) in the two cases $\Sigma^{\prime \prime}(\lambda)$ and $\Sigma^{\prime \prime}\left(s_{i} \lambda\right)$.

Identity (6) is proved as in Case I of Lemma 2.14 in [7]. Let $M_{n}^{\prime \prime}$ be the set of maximal matchings in $M_{n}$ where $\{i, i+1\}$ is an edge and let $M_{n}^{\prime}$ be the complement, that is, where the vertices $i$ and $i+1$ are not matched with each other.

For $p \in M_{n}$, let $s_{i} p$ denote the matching where we exchange the vertices $i$ and $i+1$. Note that $s_{i}$ is an involution on the set $M_{n}$ and that $M_{n}^{\prime \prime}$ is the set of fixed points of $s_{i}$. Furthermore, for a matching $p \in M_{n}^{\prime}$ we have $(-1)^{s_{i} p}=-(-1)^{p}$ and $c\left(s_{i} p, s_{i} \lambda\right)=$ $c(p, \lambda)$. Hence we obtain

$$
\sum_{p \in M_{n}^{\prime}}(-1)^{p} \cdot c(p, \lambda)+\sum_{p \in M_{n}^{\prime}}(-1)^{p} \cdot c\left(p, s_{i} \lambda\right)=0 .
$$

Again we use the unique order-preserving bijection $[n-2] \stackrel{\sim}{\rightarrow}[n]-\{i, i+1\}$. Thus if $p$ is a matching in $M_{n}^{\prime \prime}$, we can view $p-\{i, i+1\}$ as a matching on the vertex set $[n-2]$, which we will denote by $q$. This gives a bijection between $M_{n}^{\prime \prime}$ and $M_{n-2}$. Note that the two matchings $p$ and $q$ have the same sign, that is, $(-1)^{p}=(-1)^{q}$. Furthermore

$$
\begin{aligned}
c(p, \lambda)+c\left(p, s_{i} \lambda\right) & =\left(c_{2}\left(\lambda_{i}, \lambda_{i+1}\right)+c_{2}\left(\lambda_{i+1}, \lambda_{i}\right)\right) \cdot c(q, \mu) \\
& =2 \cdot \mathbb{1}_{\lambda_{i}+\lambda_{i+1}>0} \cdot c(q, \mu) .
\end{aligned}
$$

Now summing over all $p \in M_{n}^{\prime \prime}$ we obtain

$$
\begin{aligned}
\sum_{p \in M_{n}^{\prime \prime}}(-1)^{p} \cdot c(p, \lambda)+\sum_{p \in M_{n}^{\prime \prime}}(-1)^{p} \cdot c\left(p, s_{i} \lambda\right) & \\
& =2 \cdot \mathbb{1}_{\lambda_{i}+\lambda_{i+1}>0} \cdot \sum_{q \in M_{n-2}}(-1)^{q} \cdot c(q, \mu) .
\end{aligned}
$$

Identity (6) now follows by summing equations (9) and (10). 
We now include the proof of Theorem 6.4.

Proof of Theorem 6.4. We proceed by induction on $n \geqslant 1$. The induction basis is $n \leqslant 2$, and is straightforward to verify. Assume the theorem is true for $n-2 \geqslant 1$ and let us prove it for $n$. Proposition 8.1 and the induction hypothesis imply that for every $\lambda \in \mathbb{R}^{n}$ and $i \in[n-1]$ the theorem is true for $\lambda$ if and only if it is true for $s_{i} \lambda$. As the transpositions $s_{1}, s_{2}, \ldots, s_{n-1}$ generate the symmetric group $\mathfrak{S}_{n}$, we deduce that the theorem is true for $\lambda$ if and only if there exists a permutation $\tau \in \mathfrak{S}_{n}$ such that the theorem is true for $\tau \lambda$. But for every $\lambda \in \mathbb{R}^{n}$, we can find a $\tau \in \mathfrak{S}_{n}$ such that the entries of $\tau \lambda$ are in weakly increasing order. The result for $\tau \lambda$ is exactly Corollary 7.4, and so we finally deduce the result for our original $\lambda$, completing the induction step.

\section{AN EXPRESSION FOR DECREASING $\lambda$}

The base case of the proof of Theorem 6.4 is when the sequence $\lambda$ is weakly increasing. In this section we consider the other extreme, that is, when the sequence $\lambda$ is weakly decreasing. We give an expression for $S(\lambda)$ using a permutation statistic on the facets of $\Sigma(\lambda)$, that is, the set $\mathcal{A}(\lambda)$.

We begin by defining two sequences $a_{i}$ and $b_{i}$ by $a_{i}=(-1)^{\left(\begin{array}{c}i \\ 2\end{array}\right)+1}$ for $i \geqslant 1, b_{0}=$ $b_{1}=1$ and $b_{i}=2$ for $i \geqslant 2$.

LEMMA 9.1. The following identity holds:

$$
\sum_{\vec{c} \in \operatorname{Comp}(n)} a_{c_{1}} \cdot a_{c_{2}} \cdots a_{c_{k}}=(-1)^{n} \cdot b_{n},
$$

where the sum is over all compositions $\vec{c}=\left(c_{1}, c_{2}, \ldots, c_{k}\right)$ of $n$.

Proof. It is enough to see that

$$
a(x)=\sum_{n \geqslant 1} a_{n} \cdot x^{n}=\frac{-x \cdot(1-x)}{1+x^{2}}
$$

and

$$
\frac{1}{1-a(x)}=\frac{1+x^{2}}{1+x}=1+\sum_{n \geqslant 1}(-1)^{n} \cdot b_{n} \cdot x^{n} .
$$

Proposition 9.2. Let id $=12 \cdots n$ be the identity permutation. Then the following identity holds:

$$
\sum_{\sigma \in[R(\mathrm{id}), \mathrm{id}]}(-1)^{|\sigma|} \cdot(-1)^{g(\sigma)}=(-1)^{n} \cdot b_{n} .
$$

Proof. The interval $[R(\mathrm{id}), \mathrm{id}] \in \Sigma(\lambda)$ is isomorphic to the poset $\operatorname{Comp}(n)$. Hence we view the elements of this interval as compositions $\vec{c}=\left(c_{1}, c_{2}, \ldots, c_{k}\right)$ of $n$ where $\sigma$ is the ordered partition $\sigma=\left(C_{1}, C_{2}, \ldots, C_{k}\right)$ and $C_{i}$ is the interval $\left[c_{1}+c_{2}+\cdots+c_{i-1}+\right.$ $\left.1, c_{1}+c_{2}+\cdots+c_{i}\right]$. Note that $\left|C_{i}\right|=c_{i}$. Now the sum is given by

$$
\begin{aligned}
\sum_{\vec{c} \in \operatorname{Comp}(n)}(-1)^{k} \cdot \prod_{i=1}^{k}(-1)^{\left(\begin{array}{c}
c_{i} \\
2
\end{array}\right)} & =\sum_{\vec{c} \in \operatorname{Comp}(n)} \prod_{i=1}^{k}(-1)^{\left(\begin{array}{c}
c_{i} \\
2
\end{array}\right)+1} \\
& =\sum_{\vec{c} \in \operatorname{Comp}(n)} \prod_{i=1}^{k} a_{c_{i}}=(-1)^{n} \cdot b_{n},
\end{aligned}
$$

where the last equality is by Lemma 9.1 . 
For a permutation $\tau$ with descent composition $\left(c_{1}, c_{2}, \ldots, c_{k}\right)$ define

$$
b(\tau)=\prod_{i=1}^{k} b_{c_{i}}
$$

In other words, $b(\tau)$ is 2 to the power of the number of maximal ascent runs in $\tau$ which have size greater than or equal to 2 .

THEOREM 9.3. When $\lambda \in \mathbb{R}^{n}$ is such that $\lambda_{1} \geqslant \lambda_{2} \geqslant \cdots \geqslant \lambda_{n}$ the following identity holds:

$$
S(\lambda)=(-1)^{n} \cdot \sum_{\tau \in \mathcal{A}(\lambda)}(-1)^{\tau} \cdot b(\tau) .
$$

Proof. Let $\tau$ be a permutation in $\mathcal{A}(\lambda)$ with descent composition $\vec{c}=\left(c_{1}, c_{2}, \ldots, c_{k}\right)$. Let $\tau^{(i)}$ be the $i$ th descent run of the permutation $\tau$, that is, $\tau^{(i)}$ is a partial permutation and $\tau$ can be written as the concatenation of $\tau^{(1)}$ through $\tau^{(k)}$. We sum over all $\sigma$ in the interval $[R(\tau), \tau]$. In order to do so, we write $\sigma$ as a concatenation of ordered partitions $\sigma^{(1)}, \sigma^{(2)}, \ldots, \sigma^{(k)}$, where $\sigma^{(i)}$ is an ordered composition of the set of elements of $\tau^{(i)}$. The ordered partition $\sigma^{(i)}$ belongs to the interval $\left[R\left(\tau^{(i)}\right), \tau^{(i)}\right]$ in the ordered partition lattice defined on the elements of $\tau^{(i)}$.

Let $\vec{d}=\left(d_{1}, d_{2}, \ldots, d_{m}\right)$ be the block sizes of $\sigma$, that is, $\operatorname{type}(\sigma)=\vec{d}$. Note that $\vec{d} \leqslant \vec{c}$ in the poset $\operatorname{Comp}(n)$. Hence we write $\vec{d}$ as the concatenation $\vec{d}^{(1)} \circ \vec{d}^{(2)} \circ \cdots \circ \vec{d}^{(k)}$ where type $\left(\sigma^{(i)}\right)=\vec{d}^{(i)}$.

Rewrite the sum over the elements in the interval $[R(\tau), \tau]$ as follows:

$$
\begin{aligned}
\sum_{\sigma \in[R(\tau), \tau]}(-1)^{|\sigma|} \cdot(-1)^{g(\sigma)} & =\sum_{\sigma \in[R(\tau), \tau]}(-1)^{f(\sigma)} \cdot(-1)^{|\sigma|+\sum_{j=1}^{m}\left(\begin{array}{c}
d_{j} \\
2
\end{array}\right)} \\
& =(-1)^{\tau} \cdot \sum_{\sigma \in[R(\tau), \tau]} \prod_{j=1}^{m} a_{d_{j}} \\
& =(-1)^{\tau} \cdot \prod_{i=1}^{k} \sum_{\sigma^{(i)} \in\left[R\left(\tau^{(i)}\right), \tau^{(i)}\right]} \prod_{j=1}^{\left|\vec{d}^{(i)}\right|} a_{d_{j}^{(i)}}
\end{aligned}
$$

By Proposition 9.2 the sum is $(-1)^{c_{i}} \cdot b_{c_{i}}$. Finally, the product is $(-1)^{n} \cdot(-1)^{\tau}$. $b(\tau)$. By using the decomposition of $\Sigma(\lambda)$ in Proposition 4.6 and summing over all permutations $\tau$ in $\mathcal{A}(\lambda)$, the result follows.

Acknowledgements. We thank the two referees for their comments and suggestions on the exposition. We would also like to thank the École Normale Supérieure de Lyon (ÉNS de Lyon) for its hospitality and support to the second author during the academic year 2017-2018 and to the first and third author during one week visits to ÉNS de Lyon. The first and third authors also thank the Institute for Advanced Study in Princeton for hosting a research visit in Summer 2018.

\section{REFERENCES}

[1] Anders Björner, Shellable and Cohen-Macaulay partially ordered sets, Trans. Amer. Math. Soc. 260 (1980), no. 1, 159-183.

[2] - Some combinatorial and algebraic properties of Coxeter complexes and Tits buildings, Adv. in Math. 52 (1984), no. 3, 173-212.

[3] Anders Björner and Michelle L. Wachs, Shellable nonpure complexes and posets. I, Trans. Amer. Math. Soc. 348 (1996), no. 4, 1299-1327.

[4] Richard Ehrenborg and Dustin Hedmark, Filters in the partition lattice, J. Algebraic Combin. 47 (2018), no. 3, 403-439. 
[5] Richard Ehrenborg and JiYoon Jung, The topology of restricted partition posets, J. Algebraic Combin. 37 (2013), no. 4, 643-666.

[6] Robin Forman, A user's guide to discrete Morse theory, Sém. Lothar. Combin. 48 (2002), Art. B48c, 35

[7] Rebecca A. Herb, Characters of averaged discrete series on semisimple real Lie groups, Pacific J. Math. 80 (1979), no. 1, 169-177.

[8] Sophie Morel, Cohomologie d'intersection des variétés modulaires de Siegel, suite, Compos. Math. 147 (2011), no. 6, 1671-1740.

[9] Richard P. Stanley, Combinatorics and commutative algebra, second ed., Progress in Mathematics, vol. 41, Birkhäuser Boston, Inc., Boston, MA, 1996.

[10] Enumerative combinatorics. Volume 1, second ed., Cambridge Studies in Advanced Mathematics, vol. 49, Cambridge University Press, Cambridge, 2012.

Richard Ehrenborg, University of Kentucky, Department of Mathematics, Lexington, KY 40506, USA

E-mail : richard.ehrenborg@uky.edu

Sophie Morel, Princeton University, Department of Mathematics, Princeton, NJ 08540, USA

E-mail : smorel@math.princeton.edu

Margaret ReAdDy, University of Kentucky, Department of Mathematics, Lexington, KY 40506 , USA

E-mail : margaret.readdy@uky.edu 